\title{
Myoclonic seizures in a preterm baby: is this a presentation of venlafaxine withdrawal?
}

\author{
Althaf Ansary $^{1}, \mathrm{MD}, \mathrm{MRCPCH}$, Samuel Ibhanesebhor ${ }^{2}$, MRCP, FRCPCH, Chikkanayakanahalli Manjunatha ${ }^{2}$, MRCP, FRCPCH
}

\begin{abstract}
Venlafaxine, a serotonin and norepinephrine reuptake inhibitor, is increasingly used in pregnant women with pre-existing depression who require continued treatment. However, its in utero effects on the developing fetus are not clear. Herein, we report the unusual presentation of venlafaxine withdrawal in a female preterm baby of 29 weeks gestation, who presented with myoclonic seizures on her second day of life. The seizures were confirmed using amplitude-integrated electroencephalography, and other possible causes of neonatal seizures were excluded. The baby responded to treatment with phenobarbitone and phenytoin. Magnetic resonance imaging of her brain was unremarkable at corrected gestational age of 39 weeks and 2 days. On follow-up at the corrected age of five months, she was well and developing normally with no further seizures. To the best of our knowledge, this is the first report of seizures in a preterm baby resulting from maternal venlafaxine use.
\end{abstract}

Keywords: amplitude-integrated electroencephalography, myoclonic seizures, neonatal abstinence syndrome, preterm neonate, venlafaxine

\section{INTRODUCTION}

Depression during pregnancy is common and can result in serious consequences for the mother, the newborn and their family. Adequate treatment of depression during pregnancy is of great importance for maternal, fetal and neonatal health. ${ }^{(1)}$ Venlafaxine, a serotonin and norepinephrine reuptake inhibitor, and selective serotonin reuptake inhibitors (SSRIs) are commonly used antidepressants in the general population; ${ }^{(2)}$ these drugs are not uncommonly continued into the antepartum period when women with pre-existing depressive illness conceive. However, as most of these drugs cross the placenta in an erratic manner, their fetal effects are of concern. ${ }^{(3)}$ Prolonged fetal exposure to venlafaxine and SSRIs may be associated with neonatal abstinence syndrome (NAS), which affects $30 \%$ of newborns. ${ }^{(4,5)}$ Not many studies have explored the effect of venlafaxine on preterm neonates, such as the type of seizure activity produced and the electrophysiological correlation. Herein, we present what we believe is the first reported case of myoclonic seizures in a preterm neonate, in whom epileptiform activity was documented using amplitude-integrated electroencephalography (aEEG). The present case demonstrates that in utero exposure to venlafaxine can produce multifocal myoclonic seizures in babies born at 29 weeks of gestation.

\section{CASE REPORT}

A 29-week-old preterm, large-for-gestational-age (birth weight 2,335 g [> 99.6 centile]; occipital frontal circumference $31 \mathrm{~cm}$ [98 centile]) female baby was born to a 26-year-old primigravida via emergency Caesarean section due to maternal pre eclampsia. Throughout the pregnancy, the mother was on $150 \mathrm{mg}$ venlafaxine twice daily and insulin for clinical depression and poorly controlled type 1 diabetes mellitus, respectively. There was no history of smoking, or alcohol or substance abuse during the pregnancy. The mother had received magnesium sulphate infusion intrapartum and one dose of antenatal steroids prior to delivery. There was a family history of tuberous sclerosis with epilepsy in the maternal aunt.

The baby was vigorous and did not require any resuscitation at birth; cord blood gases were normal. However, the baby developed respiratory distress syndrome and needed two doses of surfactant with conventional ventilation. She was extubated to biphasic continuous positive airway pressure on Day 3. Her blood gases were normal and she remained well from a respiratory point of view, with no significant jaundice.

At 41 hours of life, the baby developed significant and persistent multifocal myoclonic jerks involving all four limbs. A recurrent high voltage pattern (lower limit $>10 \mu \mathrm{V}$; upper margin $>50 \mu \mathrm{V}$ ) was seen on aEEG, suggestive of seizure activity (Fig. 1). She was tremulous and extremely agitated, but neurological examination including tone and neonatal reflexes were appropriate for gestational age. There were no dysmorphic features or neurocutaneous markers, and the rest of the examination was unremarkable. Full blood count, blood cultures, and C-reactive protein, serum glucose and electrolyte (including magnesium) levels were all normal. Serum calcium, which was initially low $(1.81 \mathrm{mmol} / \mathrm{L})$, was

\footnotetext{
${ }^{1}$ Department of Neonatology, Royal Hospital for Sick Children (Yorkhill) Glasgow, ${ }^{2}$ Department of Neonatology, Wishaw General Hospital, Wishaw, United Kingdom Correspondence: Dr Althaf Ansary, Registrar, Department of Neonatology, Royal Hospital for Sick Children (Yorkhill), Dalnair Street, Glasgow G3 8SJ, United Kingdom. dralthaf_ansari@yahoo.com
} 


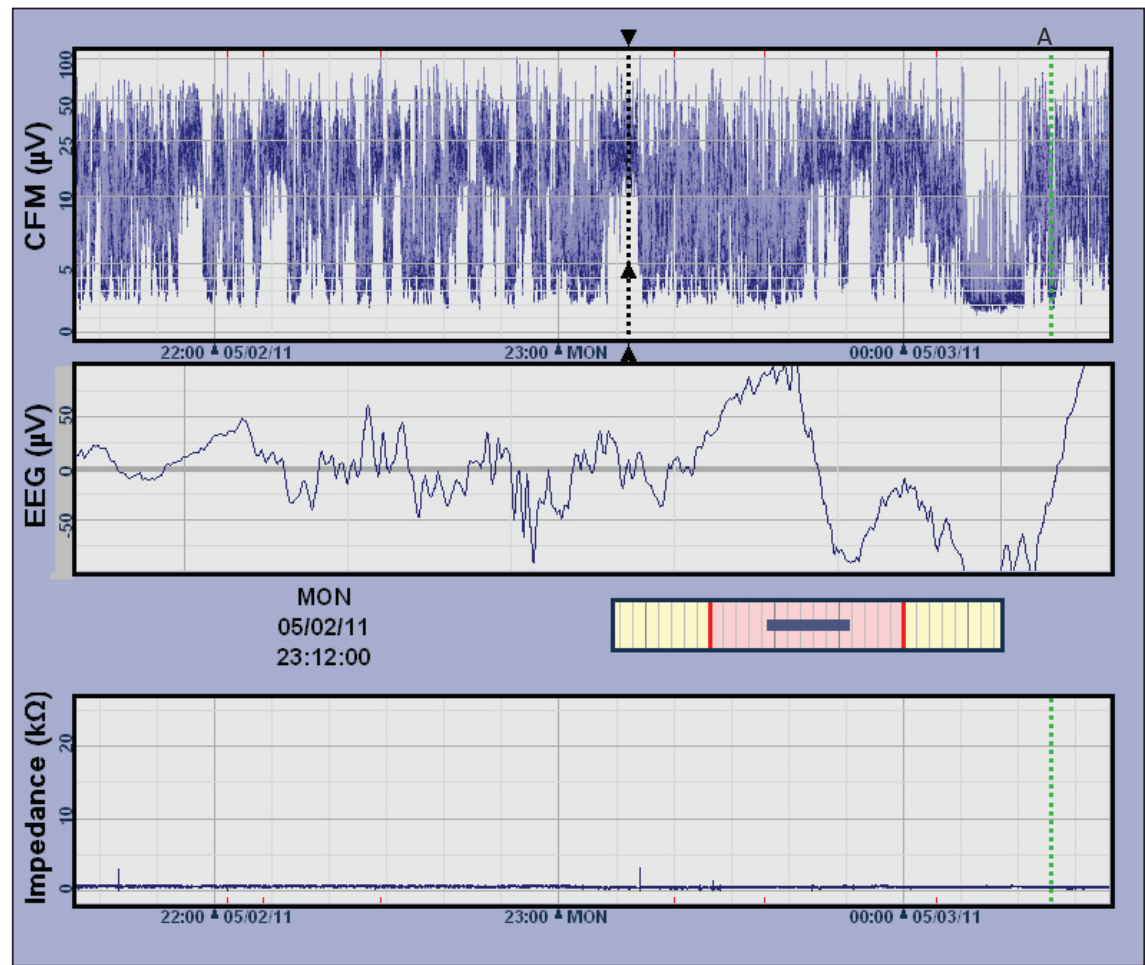

Fig. 1 Image of the cerebral function monitor (CFM) recording shows high-voltage amplitudeintegrated electroencephalography (aEEG) tracing (labelled as 'CFM' in the image). The sharp wave tracing on EEG corresponds with high voltage tracing on aEEG (dashed black line). Documentation of care procedure is marked as ' $A$ ' (dashed green line).

promptly corrected. However, treatment did not resolve the seizures. Cranial ultrasonography and cerebrospinal fluid analysis were unremarkable. Investigations for inborn errors of metabolism (IEM) were also unremarkable.

The baby was treated with two doses of intravenous phenobarbitone $(20 \mathrm{mg} / \mathrm{kg}$ and $10 \mathrm{mg} / \mathrm{kg})$, but the clinical seizures and electrophysiological abnormalities on aEEG persisted. She was then given a loading dose of intravenous phenytoin $(20 \mathrm{mg} / \mathrm{kg}$ ) and started on a maintenance dose of phenobarbitone $(2.5 \mathrm{mg} / \mathrm{kg}$ once daily). The seizures gradually settled by Day 9 , and phenobarbitone was discontinued on Day 10, with no recurrence of seizures. She was discharged on Day 59 (corrected gestational age of 38 weeks and 2 days). Magnetic resonance imaging of her brain at the corrected gestational age of 39 weeks and 2 days was unremarkable. A review conducted at the corrected age of five months found that the baby's development was satisfactory, with an unremarkable neurological examination.

\section{DISCUSSION}

The clinical presentation of drug withdrawal in neonates due to in utero venlafaxine exposure is varied. Premature birth may decrease the extent of the symptoms as there is a decrease in the duration of drug exposure prior to delivery. ${ }^{(6)}$ While there are other reports of neonatal problems secondary to maternal venlafaxine, ${ }^{(7-9)}$ only a few focused on seizure activity. We found reports of three cases of neonatal seizures as a result of in utero venlafaxine exposure; however, all three were observed in term neonates. ${ }^{(10,11)}$ To the best of our knowledge, the present case report is the first report of seizures in a preterm baby due to maternal venlafaxine use.

In the three previously reported cases of neonatal seizures, ${ }^{(10,11)}$ two neonates presented with extensor limb posturing and one with multifocal myoclonic seizures. In the present case, the preterm neonate presented with multifocal myoclonic seizures. Myoclonic seizures are the least common type of neonatal seizures, and they often carry the worst prognosis in terms of neurodevelopmental outcome and seizure recurrence. ${ }^{(12)}$ However, both the preterm neonate reported in the present case and the previously reported term neonate with myoclonic seizures responded to treatment with phenobarbitone, with normal neurodevelopmental outcome and no recurrence of seizures on follow-up. As the strongest predictor of outcome in neonatal seizures is the underlying neuropathology, accurately determining the neuropathology will enable the institution of appropriate therapy, which will lead to improved prognosis. ${ }^{(13)}$ When neonatal seizures due to withdrawal from maternal venlafaxine are identified and treated promptly, the affected neonates seem to have a favourable outcome, as evidenced by the present case and that previously reported.

Common causes of neonatal seizures include hypoxic ischaemic encephalopathy, intracranial haemorrhage, meningoencephalitis, metabolic causes (e.g. hypoglycaemia, electrolyte imbalance and IEM), developmental defects, polycythemia and NAS. ${ }^{(12)}$ In the present case, all investigations 
considered in a neonate with seizures including cranial imaging, investigations for IEM, and blood sugar, haematocrit, serum electrolyte and cerebrospinal fluid examinations were normal except for serum calcium level, which was initially low but was promptly corrected with no effect on the symptoms. Magnetic resonance imaging performed on the neonate later at term did not show any developmental disorder of the brain. As there was a clear history of maternal venlafaxine use and observed subsequent withdrawal symptoms, including seizures, a diagnosis of NAS was considered in the present case.

The diagnoses of neonatal seizures were made clinically in all of the previously reported cases except one; in that one case, the evidence of underlying seizure activity was recorded using conventional EEG. ${ }^{(11)}$ The present case is the first reported case of epileptic activity in a baby with neonatal drug withdrawal due to in utero venlafaxine exposure, documented using aEEG. Normative values for aEEG background activity at different gestational ages have previously been published. ${ }^{(14,15)}$ The practical advantage of aEEG over conventional EEG in sick full-term and premature infants is that aEEG can be well integrated into the routine care of any newborn infant admitted into a neonatal intensive care unit, as exemplified in the present case.

Seizures are most often represented on aEEG as a sudden narrowing and rise in the band of activity (i.e. a rapid rise in both the lower and upper margins of the trace). This is because the brain displays a high level of continuous activity during seizures. Arousal during care procedures often appear as an artifact, with a transient rise in the aEEG background. This artifact may look similar to epileptic seizure activity. In the present case, all care procedures were documented on the traces to facilitate correct interpretation. Status epilepticus may appear either as a 'saw-tooth' pattern or as a continuously raised background pattern. Raw EEG recording, which is available simultaneously on cerebral function monitor (CFM) machines, is very useful in assisting accurate interpretation of the patterns observed.(16) In the present case, concurrent EEG recording on CFM showed sharp spike wave tracings that corresponded to the high voltage tracings of the aEEG (Fig. 1).

The use of antidepressant medication during pregnancy is unlikely to decrease in the near future. Therefore, consideration should be given to tapering the dose of all antidepressants, including venlafaxine, in the third trimester to decrease the risk of withdrawal symptoms in neonates. ${ }^{(1)}$ In the present case, the baby was born prematurely, before such an intervention could have been considered.

In conclusion, a high index of suspicion and an abnormal aEEG pattern may help in the identification of seizure activity in a preterm baby with neonatal drug withdrawal. Supportive management after the exclusion of other causes of neonatal seizures is advised until the resolution of seizures. With proper management, the neurodevelopmental outcome of neonatal seizures caused by venlafaxine withdrawal appears favourable, as evidenced in previous reports and the present case. There is a need for parents and health professionals to be educated on the possible risk of withdrawal in neonates. There is also a pressing need for further investigations on the long-term developmental outcomes of infants exposed to venlafaxine, an increasingly prescribed antidepressant. The importance of individualised risk-benefit analysis of maternal antidepressant use with respect to the drug's potential impact on the developing fetus cannot be overemphasised. Interventions to prevent neonatal drug withdrawal, such as the tapering of medication doses in the third trimester of pregnancy, should be carefully considered.

\section{ACKNOWLEDGEMENT}

The authors are grateful to the parents of the neonate reported in the present case report for their consent to the publication of this case report in a medical journal.

\section{REFERENCES}

1. Patil AS, Kuller JA, Rhee EH. Antidepressants in pregnancy: a review of commonly prescribed medications. Obstet Gynecol Surv 2011; 66:777-87.

2. Brown CS. Depression and anxiety disorders. Obstet Gynecol Clin North Am 2001; 28:241-68.

3. Hostetter A, Ritchie JC, Stowe ZN. Amniotic fluid and umbilical cord blood concentrations of antidepressants in three women. Biol Psychiatry 2000; 48:1032-4.

4. Levinson-Castiel R, Merlob P, Linder N, Sirota L, Klinger G. Neonatal abstinence syndrome after in utero exposure to selective serotonin reuptake inhibitors in term infants. Arch Pediatr Adolesc Med 2006; 160:173-6.

5. Oberlander TF, Misri S, Fitzgerald CE, et al. Pharmacologic factors associated with transient neonatal symptoms following prenatal psychotropic medication exposure. J Clin Psychiatry 2004; 65:230-7.

6. Klinger G, Merlob P. Selective serotonin reuptake inhibitor induced neonatal abstinence syndrome. Isr J Psychiatry Relat Sci 2008; 45:107-13.

7. Einarson A, Fatoye B, Sarkar M, et al. Pregnancy outcome following gestational exposure to venlafaxine: a multicenter prospective controlled study. Am J Psychiatry 2001; 158:1728-30.

8. De Moor RA, Mourad L, ter Haar J, Egberts AC. [Withdrawal symptoms in a neonate following exposure to venlafaxine during pregnancy]. Ned Tijdschr Geneeskd 2003; 147:1370-2. Dutch.

9. Sanz EJ, De-las-Cuevas C, Kiuru A, Bate A, Edwards R. Selective serotonin reuptake inhibitors in pregnant women and neonatal withdrawal syndrome: a database analysis. Lancet 2005; 365:482-7.

10. Pakalapati RK, Bolisetty S, Austin MP, Oei J. Neonatal seizures from in utero venlafaxine exposure. Paediatr Child Health 2006; 42:737-8.

11. Hoppenbrouwers CJ, Bosma J, Wennink HJ, et al. Neonatal seizures on EEG after in utero exposure to venlafaxine. Br J Clin Pharmacol 2010; 70:454-6.

12. Sankar MJ, Agarwal R, Aggarwal R, Deorari AK, Paul VK. Seizures in the newborn. Indian J Pediatr 2008; 75:149-55.

13. Volpes JJ. Neonatal Seizures. In: Neurology of the newborn. 5th ed. Philadelphia: Saunders Elsevier; 2008, p223-30.

14. Thornberg E, Thiringer K. Normal patterns of cerebral function monitor traces in term and preterm neonates. Acta Paediatr Scand 1990; 79:20-5.

15. Olischar M, Klebermass K, Kuhle S, et al. Reference values for amplitudeintegrated electroencephalographic activity in preterm infants younger than 30 weeks' gestational age. Pediatrics 2004; 113:e61-6.

16. de Vries LS, Hellström-Westas L. Role of cerebral function monitoring in the newborn. Arch Dis Child Fetal Neonatal Ed 2005; 90:F201-7. 